\title{
Le Canada et les Roms : gouverner l'inconnaissable
}

\author{
Mark B. Salter
}

Traducteur : Vincent Laflamme (depuis l'anglais)

\section{(2) OpenEdition \\ Journals}

\section{Édition électronique}

URL : http://journals.openedition.org/conflits/18153

DOI : $10.4000 /$ conflits. 18153

ISSN : $1777-5345$

Éditeur :

CCLS - Centre d'études sur les conflits lilberté et sécurité, L'Harmattan

Édition imprimée

Date de publication : 5 septembre 2011

Pagination : 208-212

ISBN : 978-2-296-56086-4

ISSN : 1157-996X

\section{Référence électronique}

Mark B. Salter, «Le Canada et les Roms : gouverner l'inconnaissable », Cultures \& Conflits [En ligne],

81-82 | Printemps/Été 2011, mis en ligne le 05 septembre 2012, consulté le 30 mars 2021. URL :

http://journals.openedition.org/conflits/18153 ; DOI : https://doi.org/10.4000/conflits.18153 


\title{
Le Canada et les Roms : gouverner l'inconnaissable *
}

\author{
Mark B. SALTER
}

Mark B. Salter est Professeur associé à l'École d'Études Politiques de l'Université d'Ottawa. Il est auteur de l'ouvrage Rights of Passage: the passport in international relations (Lynne Rienner, 2003) et a dirigé Mapping Transatlantic Security Relations (Routledge, 2010).

\begin{abstract}
ux yeux du Conseil de l'Europe et de l'Union européenne, de même que - 1 our plusieurs États européens, le groupe connu sous le nom de «Roms » pose un problème de nature politique : ce groupe remet en question les conceptions d'identité nationale, de lieu, d'appartenance, de territoire, de citoyenneté et de droits. Il est difficile de définir de façon empirique la « communauté » rom, que ce soit relativement à la taille de sa population (entre huit et douze millions), à sa localisation (partout en Europe), à ses caractéristiques ethniques, ou encore à l'existence d'une culture ou d'une langue commune (par exemple, si plusieurs dialectes Roms sont mutuellement compréhensibles, d'autres ne le sont pas). Cependant, d'un pays à l'autre, les différentes communautés identifiées en tant que Roms souffrent manifestement de discriminations ethniques et raciales; de difficultés aux chapitres de l'intégration, de la mobilité et de l'assimilation ; en plus d'être souvent victimes d'attaques violentes. Il est possible de se référer à la Décennie pour l'intégration des Roms (2005-2015) ${ }^{1}$, aux travaux de recherche, aux plans d'action et aux politiques de sensibilisation pour définir, comprendre et gérer la question, et ainsi confirmer l'existence de la problématique.
\end{abstract}

Contrairement au cas européen, les Roms ne posent pas de problème au Canada dans la mesure où ils ne constituent pas une catégorie ou une population à l'égard de laquelle le gouvernement canadien exprime un besoin de gou-

*. Traduit de l'anglais par Vincent Laflamme.

1. En 2005, douze États, essentiellement d'Europe de l'Est, ont proclamé, avec le soutien de la Banque mondiale, du Programme des Nations unies pour le développement (PNUD) ou encore de l'Organisation pour la sécurité et la coopération en Europe (OSCE), 2005-2015 «Décennie de l'intégration des Roms ». L'accent a été mis sur quatre domaines prioritaires : éducation, emploi, logement et santé. 
verner. La plus grande communauté Rom au Canada est située à Toronto, est comprise et présentée comme étant assimilée, et évolue sans heurt au sein d'un Canada multiculturel. Le Centre communautaire Rom de Toronto estime que la population Rom se chiffre à plus de quatre-vingt mille individus, bien que d'autres sources soutiennent que ce nombre avoisine les trente mille, mais le manque même de données indique que les Roms ne constituent pas, au Canada, une simple catégorie politique ${ }^{2}$. Il n'existe aucune statistique officielle sur la population Rom au Canada : le gouvernement ne la considère tout simplement pas comme une catégorie de population qu'il importe d'étudier. Cette différence de consensus relativement à ce qui pose ou non un problème peut nous éclairer sur la topologie d'un champ transnational des professionnels de la population et de la gestion sécuritaire ${ }^{3}$. Quoiqu'il existe un consensus au sujet de la gestion de la migration internationale, de l'utilisation de visas et d'accords avec des pays tiers pour délocaliser les frontières, de l'usage de données biométriques et de passeports pour suivre les traces des populations mobiles, dans le cas des Roms, nous pouvons voir que la composante première du champ doit être l'existence d'une épistémologie partagée relativement à ce qui est considéré ou non comme un problème.

Les Roms apparaissent seulement comme objet de contrôle lorsqu'il est question de demandes d'asile - originairement en provenance de République Tchèque, mais aussi de plus en plus en provenance de Hongrie. L'augmentation du nombre de Roms fuyant la persécution et cherchant refuge à l'étranger reflète la précarisation de la situation dans ces deux pays ${ }^{4}$. Au Canada, le traitement des demandes d'asile est effectué par la Commission de l'immigration et du statut de refugié (CISR), une instance gouvernementale semi-autonome. La CISR ne conserve aucune statistique concernant l'ethnicité ou l'identité en raison de la nature privée de ces informations. Par conséquent, nous percevons un raisonnement indirect : il y a une augmentation de demandes d'asile en provenance d'États où les Roms subissent des persécutions et dont les demandes d'asile profitent d'un haut degré d'acceptation de la part de la CISR. Cependant, du fait que les Roms n'apparaissent pas comme une population chiffrable, Citoyenneté et immigration Canada - le ministère chargé de gérer le secteur de l'immigration au Canada - connaît un problème de gouvernance : comment administrer l'incomptable ?

Bien que le visa canado-tchèque soit perçu de part et d'autre comme un objet de tensions mineures dans le contexte plus large des saines relations bila-

2. Lee R., "Roma in Canada”, Roma Community Centre, 1998. Disponible à l'adresse suivante : http://www.romatoronto.org/facts_canada.html, page consultée le 12 mai 2011.

3. Bigo D., "Globalized (in)security: the field and the ban-opticon”, in Bigo D., Tsoukala A. (eds.) Terror, Insecurity and Liberty: Illiberal Practices of Liberal Regimes after 9/11, Londres, Routledge, 2008, pp. 12-16.

4. Saunders D., “The Next Stop for the Roma: Canada?”, The Globe and Mail, 22 janvier, 2010 ; disponible à l'adresse suivante : http://www.theglobeandmail.com/news/world/the-nextstop-for-the-roma-canada/article1441459/, page consultée le 12 mai 2011. 
térales, son existence entraîne toutefois un problème à l'échelle européenne. La République Tchèque, en tant que signataire de l'accord Schengen, ne peut imposer un visa punitif aux ressortissants canadiens dans le but d'exercer une pression sur le gouvernement canadien. Le Canada apparaît sur la « liste blanche de Schengen », avec non obligation de visa, dans la mesure où - exception faite de la République Tchèque - le gouvernement canadien n'exige pas de visa de la part des pays de l'espace Schengen, et que la politique commune concernant les visas est caractérisée par la notion de réciprocité. Au mieux, il s'agit d'une réciprocité asymétrique si l'on considère que l'UE n'a pas répliqué à l'imposition canadienne d'un visa en exigeant, à son tour, des visas aux ressortissants canadiens. Parce que le prochain accord intégral entre l'UE et le Canada nécessitera la ratification des États membres - à savoir non seulement la ratification de la République Tchèque, mais également celles de la Roumanie et de la Bulgarie (pays dont les ressortissants se voient imposer l'obligation de visa par le Canada, mais qui ne sont pas encore membre de l'accord Schengen) - il est possible que cette pression structurera la position canadienne sur l'enjeu des visas.

Le gouvernement canadien gère la migration, et plus particulièrement les demandes d'asile en provenance de la République Tchèque, par le biais de politiques sur les visas et les réfugiés, ce qui comprend notamment des ententes concernant des pays tiers sûrs. Le Canada a modifié à quatre reprises sa politique sur les visas destinés à la République Tchèque - ce qui a entraîné des effets considérables. Le $1^{\mathrm{er}}$ janvier 1997, le Canada a levé l'obligation de visa, mais il a immédiatement dû composer avec une arrivée massive de demandeurs d'asile, provoquée en grande partie par la diffusion à la télévision tchèque d'une émission qui présentait l'adoucissement des exigences relatives aux demandes d'asile et le niveau de vie élevé au Canada 5. S'étant identifiés pour la plupart comme Roms, ces demandeurs d'asile fondaient leurs demandes sur la persécution dont ils étaient victimes ou, du moins, sur l'absence de protection que leur accordait l'État tchèque. Le Canada n'inclut toutefois pas la nationalité Rom dans ses statistiques, et Citoyenneté et immigration Canada - soit le ministère responsable de gérer cette question - ne possède aucun pouvoir légal pour astreindre, réguler ou gérer les mouvements migratoires de nature infranationale ou non nationale. En conséquence, bien que toutes les parties en cause reconnaissent que l'enjeu réel porte sur la vulnérabilité de la population Rom devant un État tchèque agressif ou, du moins, non-protecteur, l'exigence d'un visa pour tous les ressortissants tchèques constitue le seul instrument politique dont le ministre peut se pourvoir. Depuis, cette exigence a rendu plus difficile l'arrivée au Canada pour les Roms non définis, ce qui constitue pourtant une étape préalable à la formulation de leur demande.

5. Lefebvre S., "Roma and the Czech-Canadian visa issue, 1998-9", Contemporary Politics, 9, 3, 2003, pp.313-322. 
À deux reprises, en 1997 et en 2009, à la suite de la levée des restrictions canadiennes sur les visas, de nombreuses demandes d'asile ont été formulées par des ressortissants tchèques, et ce dès l'exemption du visa. Le ministre de la citoyenneté et de l'immigration, Jason Kenney, a fait deux déclarations publiques à ce sujet qu'il importe de souligner. En premier lieu, a-t-il affirmé, "I find it hard to believe that the Czech Republic is an island of persecution in Europe ", signifiant par là non seulement l'idée que la République Tchèque constitue un État démocratique, mais également le fait que le Protocole Aznar - qui interdit de façon efficace n'importe quelle demande d'asile de citoyens européens faite à l'endroit de tout autre pays européen - offre en définitive aux Roms la possibilité de se déplacer vers un pays européen moins dangereux ${ }^{6}$. En second lieu, le ministre Kenney a insinué que les nombreux désistements faits par des demandeurs d'asile tchèques laissaient présager l'existence de fraudes. En raison de la grande proportion d'abandons ou de désistements survenue à la suite des trois mille demandes effectuées en 2009 dès l'exemption des visas - soit près de $50 \%$-, le ministre Kenney a fait valoir que ces nombreux cas frauduleux mettaient en doute l'intégrité des procédures de même que l'équité du système. Un avocat qui a représenté quelques uns des Roms ayant abandonné leur demande avançait que le délai pour les entrevues ne permettait aucunement aux demandeurs d'asile de demeurer au Canada. Cela dit, une grande partie des demandes entendues en 2009 ont été approuvées ${ }^{7}$. Le visa a été réimposé aux ressortissants tchèques pour gérer, de façon indirecte, le défi que pose la question des Roms. Partant, la définition et la réponse qu'offre le Canada relativement à cette question demeurent indirectes : le Canada ne peut pas connâtre ni définir la population actuelle de Roms qu'il souhaite gérer, se servant ainsi de la population tchèque (ou hongroise) comme d'une procuration. Alors que le Canada ne reconnaît pas ouvertement la situation des Roms comme étant une justification appropriée en matière de demande d'asile, lorsque les équipes de la CISR et celles de Citoyenneté et immigration Canada ont visité la République tchèque pour déterminer respectivement le bien-fondé des cas de demandes l'asile et la pertinence de l'exemption des visas, elles ont, dans leurs rapports, porté une attention bureaucratique à la question des Roms ${ }^{8}$. S'exprimant toutefois au nom des élites dirigeantes, le ministre Kenney a affirmé que :

«[w]e have only one tool available to address those situations where we see an aberrant enormous wave of unfounded claims from a democratic instrument. You know what that tool is? It's a very blunt instrument - the imposition of a visa requirement for visitors. This is a blunt instrument. It undermines Canada's commercial and

6. Badiuzzaman S., "Roma asylum seekers give up on Canada", Agence France Presse, 2 novembre 2010. Disponible à l'adresse http://www.google.com/hostednews/afp/article/ALeqM5ihOrEH1ID5rYRP90reBoLUYrw CfQ?docId=CNG.ed091710b328fd92692ce44b7df6f8e1.df1, page consultée le 12 mai 2011.

7. Ibid.

8. Lefebvre S., op. cit., p. 314. 
diplomatic interests. It's a necessary tool to use in a managed immigration system but you want it only as your last resort ${ }^{9}$ ».

Si nous acceptons l'analyse de Foucault selon laquelle la limitation de l'intervention gouvernementale constitue le problème fondamental des régimes libéraux ${ }^{10}$, alors comment est-il possible que cette politique - laquelle, reconnaît le ministre Kenney, est de nature étatiste tout en étant contraire aux intérêts commerciaux et diplomatiques du gouvernement - soit aujourd'hui perçue comme la seule action gouvernementale possible ? L'adoption récente du projet de loi C-11 - la Loi sur des mesures de réforme équitables concernant les réfugiés 11 - révèle deux nouvelles voies qu'empruntera le gouvernement pour tenter de gérer cette question qui, à la base, porte sur l'inconnaissable. La première voie est celle du « pays d'origine désigné »; la seconde, celle de la prétendue «prochaine génération de visa ». La première constitue une reformulation de la classification initiale de « pays d'origine sûr », laquelle classification suggérait implicitement que les demandes en provenance des pays ainsi désignés étaient reçues avec davantage de scepticisme. Les amendements apportés au système ont simplement permis d'accélérer le processus des entrevues concernant les demandes d'asile en provenance des pays désignés. Par la seconde voie, on tente de transformer le visa en instrument politique mieux ciblé, et ce en faisant des individus, par opposition à la population nationale, le nouvel objet de gouvernance.

Le Canada ne conçoit pas les Roms comme un problème de population en soi, puisque ces derniers n'apparaissent pas comme une catégorie ou un groupe statistique. Lorsque de nombreux demandeurs d'asile formulent leur demande - évoquant pour cela la situation qu'ils subissent en tant que Roms ils deviennent dès lors, en tant que population invisible, un problème invisible, et des outils indirects sont utilisés pour les gérer.

9. Kenney J., "Opening remarks for the Honourable Jason Kenney, P.C., M.P. Minister of Citizenship, Immigration and Multiculturalism Balanced Refugee Reform Act" Citizenship and Immigration Canada, 8 avril 2010. Disponible à l'adresse : http://cic.gc.ca/english/department/media/speeches/2010/2010-04-08.asp, page consultée le 12 mai 2011.

10. Foucault M., The Birth of Biopolitics: Lectures at the Collège de France, 1978-1979 (Traduction Graham Burchell), New York, Palgrave Macmillan, 2008, p. 319.

11. Loi sur des mesures de réforme équitables concernant les réfugiés, Lois du Canada, 2010, ch. 8, Sanctionnée le 29 juin 2010, http://loislaws.justice.gc.ca/fra/LoisAnnuelles/2010_8/TexteComplet.html, page consultée le 12 mai 2011. 